\title{
Nonlinear Analysis on Traffic Flow Based on Catastrophe and Chaos Theory
}

\author{
Jian Gu and Shuyan Chen \\ School of Transportation, Southeast University, Nanjing 210008, China \\ Correspondence should be addressed to Shuyan Chen; chenshuyan@seu.edu.cn
}

Received 22 May 2014; Revised 6 October 2014; Accepted 15 October 2014; Published 27 November 2014

Academic Editor: Seenith Sivasundaram

Copyright ( $92014 \mathrm{~J}$. Gu and S. Chen. This is an open access article distributed under the Creative Commons Attribution License, which permits unrestricted use, distribution, and reproduction in any medium, provided the original work is properly cited.

\begin{abstract}
We applied catastrophe and chaos theory to analyze the traffic nonlinear characteristics of expressway condition. Catastrophe theory was generally used to explore the mathematical relationships among the traffic data collected from highway conditions, which could not be appropriate for the urban expressway conditions. Traffic flow data collected from the 3rd ring road expressway in Beijing was used to build flow-density model and speed-density Greenshields model. Then the density was discussed based on the traffic wave speed function with cusp catastrophe theory; in particular, density conditions on median lanes and shoulder lanes were deeply discussed. Meanwhile the chaotic characteristics were analyzed based on the traffic temporal sequence data collected from 29 detectors located at the 3rd ring road expressway, and C-C method was used to reconstruct the phase space and the largest Lyapunov exponents were estimated by Wolf method and the small data sets method. The results indicated that the traffic operation catastrophe density on the median lanes was a bit higher than that on the shoulder lanes; additionally chaotic characteristics obviously existed in the local corridor composed of 29 detectors in the 3rd ring road expressway traffic flow system.
\end{abstract}

\section{Introduction}

This paper is to analyze traffic nonlinear characteristics by using catastrophe theory and chaos theory. Traffic can be considered as a nonlinear system, and catastrophe and chaotic phenomena have been observed, which are supported by catastrophe theory and chaos theory, respectively. Catastrophe theory set up by René Thom is a branch of bifurcation theory in the study of dynamical systems, and it may conceptually explain the manner in which a discontinuity or "jump" happens. Chaos theory studies the behavior of dynamical systems that are highly sensitive to initial conditions, a response popularly referred to as the butterfly effect, and the most striking feature of chaos is the unpredictability of its future; a tiny difference or even a cut-off error will be blown up quickly and results in a big difference in the near future. The relationships between catastrophe theory and chaos theory rely on a bifurcation, which displays changes of topological structure in phase diagram when control parameters change. After the bifurcation happens, discontinuous transition will exist among system situations, which may be called as the catastrophe. Bifurcation occurs continually, and the system ultimate state will be discussed eventually by the chaotic theory. However, in dense traffic, small disturbances such as the acceleration or deceleration of one vehicle could be passed over or amplified along the line, and it might seem to be sensitive to the initial conditions which caused chaos. Thus it is difficult to build dynamic function considering complex factors in traffic flow system, so the temporal sequence analysis will be used.

Traffic nonlinear system has been studied by a vast variety of microscopic and macroscopic methods, besides catastrophe theory and chaos theory. And microscopic modeling has inherent superiority, of which agent-based approach can delve into individual-level dynamics and provides insight into the evolutionary process. Vehicles travelling in the lanes interact with each other, which can be seen as group interactions problems and solved by games methods and others. Perc studied the impact of evolutionary games on traffic flow with the discrete cellular automation Biham-MiddletonLevine (BML) model, and the work induced a premature seizure of traffic flow by a substantially lower initial density 
of agents as in the original case [1]. Additionally, Perc et al. reviewed the works on evolutionary games incorporating coevolutionary rules and particularly highlighted the study of evolutionary dynamics of group interactions on structured populations [2, 3], enriching the theoretical methods to certain problems. Cell transmission model (CTM) and the agent-based methods had been applied to elaborate the emergence phenomena in traffic system, such as the cell transmission model had been applied to ensure first-infirst-out (FIFO) dynamic traffic assignment and agent-based simulator had been used to study congestion pricing schemes $[4,5]$.

Agent-based methods can effectively understand vehicles operation and pedestrian behavior; however, in the fields of traffic management, macroscopic methods own exclusive advantages on analyzing traffic characteristics; for example, Greenshields model is common applied in traffic practice. Tadaki et al. analyzed the phenomenological features of the real traffic flow observed at the upper stream of a tunnel, and temporal sequences and statistical analyses were used to discuss the phase transition between freely moving and congested states [6]. Geroliminis and Daganzo analyzed a field experiment using a combination of fixed detectors and floating vehicles and revealed that a macroscopic fundamental diagram (MFD) linking space-mean flow, density, and speed exists on a large urban area [7]; additionally Geroliminis and Sun investigated what were the properties that a network should satisfy, so that an MFD with low scatter existed and the results indicated that the spatial distribution of vehicle density in the network was one of the key components that affected the scatter of an MFD and its shape [8]. Daganzo et al. had provided an explanation on networks jam due to multiple overlapping routes with MFD [9].

Among the macroscopic methods, catastrophe theory and chaotic theory are adept at indicating complex operation of nonlinear traffic system. Hall et al. analyzed the occurrences of gaps in freeway operation data and the accompany jumps in the behavior of traffic parameters with catastrophe theory [10-12], Zhang and Pei discussed the application of cusp catastrophe theory in traffic flow prediction [13], Guo et al. discussed traffic models with cusp catastrophe theory [14], and Papacharalampous and Vlahogianni proposed a framework based on stochastic cusp catastrophe theory to model microscopic freeway traffic flow considering that each driver may shift his/her behavior and behave aggressively or timidly during driving [15].

Chaotic characteristics in traffic flow system have been also confirmed. Zhang and Jarrett examined the chaotic traffic by car-following model [16]. Nagatani used the lattice hydrodynamic model to take into account the passing effect in one-dimensional traffic flow and found that the chaotic jams appear when the passing constant was larger than 0.1 [17]. Li proposed a class of discrete models derived from nonequilibrium continuum models to identify and predict the self-organized vehicle clustering and the transition to chaos in traffic flow [18]. Shang et al. analyzed traffic data collected from Beijing Xizhimen, and the results indicated that chaotic characteristics existed obviously and speed was predicted [19]. Lo and Cho applied traffic three-parameter model and fundamental diagram models to analyze traffic chaos, and several chaotic control strategies were discussed [20]. Lan et al. discussed traffic dynamical evolution by the largest Lyapunov exponent and correlation dimension in the traffic state reconstruction spaces [21].

Conclusions from literatures above are mainly inferred from highway condition; however it is different from urban expressway condition, which has smaller distance between ramps, and vehicles could be easily influenced when travelling near the ramps. Traffic operation of the middle, median, and shoulder lanes would be disturbed as well; thus, the conclusions did not satisfy the expressway condition. Reasonable analysis on expressway traffic flow system is necessary to formulate traffic management and control strategy.

In addition, conventional models based on the typical traffic fundamental diagram could evolve traffic parameters better. However, complex and stochastic factors, such as driver behavior, weather, and environment, would bring the difficulty to build precise models. For example, discontinuous or jump phenomena have been observed in the traffic flow data, which is hard to be explained by the three-parameter traffic fundamental continuous models in two-dimensional plane. On the other side, chaos makes it unpredictable for traffic forecasting.

Although theoretical analysis has been used to comprehend traffic nonlinear system, actual data is less used to motivate the theoretical treatment. The present paper extends the theoretical analysis by applying macroscopic models such as catastrophe theory and chaos theory, in which cusp catastrophe model was used to analyze expressway traffic nonlinear characteristics and the parameter of density was discussed deeply. Meanwhile expressway traffic chaotic characteristics were investigated in detail based on traffic temporal sequence data.

This paper consists of four sections. Section 2 introduces the basic method of cusp catastrophe theory with traffic flow model and chaotic theory. Section 3 contains the data implementation including data description and parameter calculation. Section 4 provides the conclusion.

\section{Methodology}

2.1. Cusp Catastrophe Applied to Macroscopic Traffic. According to stability theory and bifurcation theory, René Thom set up the catastrophe theory based on discontinuous research which can explain the discontinuous transition of a system between equilibrium states. Seven types of catastrophe models were induced from the singularities of gradient system by building relations with differential equations and functions. Dynamical system of traffic parameters also represents the same five characteristics in the fields of bimodality, hysteresis, inaccessible behavior, catastrophe, and divergence, so the traffic model problem can be solved by catastrophe theory, and one of the models, cusp catastrophe model, is selected for 
analyzing due to its wide use. And its equations are shown as follows:

potential function:

$$
V(x)=x^{4}+b x^{2}+c x
$$

balance curved surface:

$$
4 x^{3}+2 b x+c=0
$$

catastrophe set:

$$
\begin{aligned}
& 4 x^{3}+2 b x+c=0 \\
& 12 x^{2}+2 b=0
\end{aligned}
$$

where $x$ denotes state variable and $b, c$ denote control factors (bifurcation/splitting factor and normal/asymmetry factor, resp.).

The three macroscopic measures of the state of a given traffic stream, flow $Q$, space mean speed $V$, density $K$, are used to evolve traffic operation, and the basic relationship among them is given as $Q=V * K$. Meanwhile, generalized car-following model, discussed in May and Keller's work [22], is used as (4), where $V_{f}$ denotes the free flow velocity value and $K_{j}$ denotes congested density:

$$
V^{1-m}=V_{f}^{1-m}\left[1-\left(\frac{K}{K_{j}}\right)^{l-1}\right] .
$$

When $m=0, l=3$, (4) can be the widely used model as follows:

$$
V=V_{f}\left[1-\left(\frac{K}{K_{j}}\right)^{2}\right] .
$$

The following is the first differential of (5) on $K$ :

$$
\frac{d V}{d K}=-2 \frac{V_{f}}{K_{j}}\left(\frac{K}{K_{j}}\right) .
$$

Traffic shock wave $V_{\omega}$ is deduced as

$$
V_{\omega}=\frac{\Delta Q}{\Delta K}=\frac{d Q}{d K}=K \frac{d V}{d K}+V=K \frac{d V}{d K}+\frac{Q}{K} .
$$

Traffic shock wave could be rewritten as follows based on (6) and (7):

$$
\begin{gathered}
V_{\omega}=\frac{Q}{K}-2 K \frac{V_{f}}{K_{j}}\left(\frac{K}{K_{j}}\right), \\
K^{3}+\frac{K_{j}^{2}}{2 V_{f}} \cdot V_{\omega} \cdot K-\frac{K_{j}^{2}}{2 V_{f}} \cdot Q=0 .
\end{gathered}
$$

Equation (9) is similar to the cusp catastrophe balanced curved surface function and the potential function may be designed as

$$
P\left(K, V_{\omega}, \mathrm{Q}\right)=\frac{1}{4} K^{4}+\frac{1}{4} \frac{K_{j}^{2}}{V_{f}} \cdot V_{\omega} \cdot K^{2}-\frac{K_{j}^{2}}{2 V_{f}} \cdot \mathrm{Q} \cdot K
$$

Then the critical wave speed and density are deduced as (11) and (12) as discussed in Guo et al.'s work [4]:

$$
\begin{gathered}
V_{\omega}=-\left(\frac{27 V_{f}}{2 K_{j}^{2}} \cdot Q^{2}\right)^{1 / 3}, \\
K_{\text {cusp }}=\left(\frac{2 K_{j}^{2}}{V_{f}} \cdot Q\right)^{1 / 3} .
\end{gathered}
$$

From (12), the catastrophe critical density $K_{\text {cusp }}$ has a relation with the traffic flow $Q$, and when vehicles pass by, there will be a maximum volume, $Q_{\max }$, which is selected to calculate the $K_{\text {cusp }}$ :

$$
K_{\text {mcusp }}=\left(\frac{2 K_{j}^{2}}{V_{f}} \cdot Q_{\max }\right)^{1 / 3}
$$

2.2. Chaos Identify Methods. In traffic flow when the control parameter of Logistic map equals 3.5 and 4, the traffic flow is chaotic; however, in dense traffic, small disturbances such as the acceleration or deceleration of one vehicle could be passed over or amplified along the line, and it might seem to be sensitive to the initial conditions which caused chaos. Thus it is difficult to build dynamic function considering complex factors in traffic flow system, so the time series analysis will be used, which is ubiquitous in the chaos analysis [23].

Chaotic characteristics can be identified from time series both on qualitative and quantitative aspects. In the qualitative aspect, chaotic characteristic can be intuitively identified from the diagrams, distinctly presenting dynamic characteristics. Common methods such as analyzing power spectrum diagram and wavelet decomposition diagram can be used. In the power spectrum diagram the spectrum is continuous but not flat with noise and broad peak, and in the wavelet decomposition diagram the components scatter in frequency domain presenting self-similarity at each decomposition level.

In the quantitative aspect, calculation on singular attractor characteristic parameters depicting the rate of expansion or contraction in the spaces can help identify chaos in theory, including Lyapunov exponent, correlation dimension, and Kolmogorov entropy, among which the estimation of Lyapunov exponent is relatively easy to compute, accurate, and widely used by Wolf method or small data sets method $[24,25]$, and the positive largest Lyapunov exponent can yield evidence for the presence of deterministic chaos in the observed data time series [19].

In addition, reconstruction of phase space is the important precondition, which consists of the determination of the delay time $\tau$ and the embedding dimension $m$. Autocorrelation function method, mutual information, and C-C method are usually applied, especially using $\mathrm{C}-\mathrm{C}$ method which can synchronously estimate the delay time and the embedding dimension, which used the correlation integral in the BDS statistic. Both the reconstruction parameters $\tau$ and $m$ must be determined from the actual data [26]. 


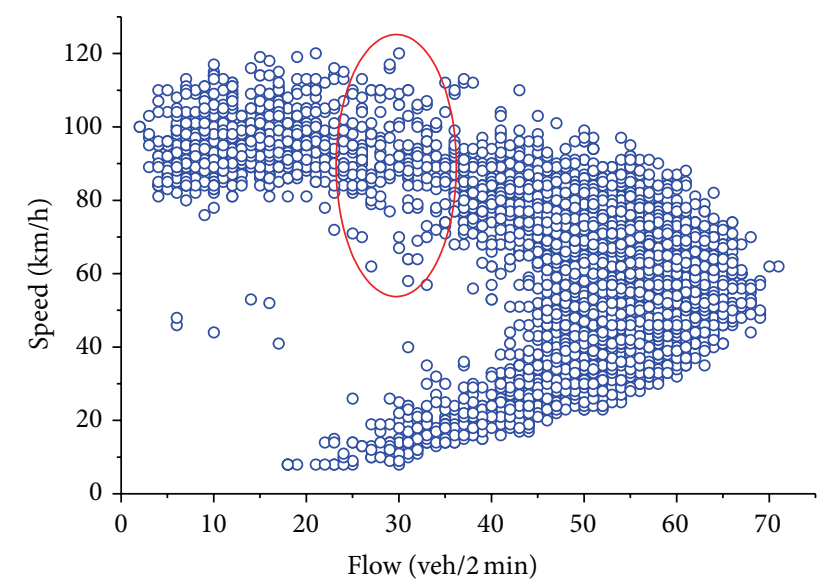

FIGURE 1: Scatter illustration of speed-flow from RTMS 3060. Small blue circles stand for the relation between speed and flow, and the red ellipse zone stands for the joint zone linked with free and congested traffic situations. Symbols show conditions of discontinuity.

\section{Implementation and Results}

An empirical study was implemented as follows. Actual data set collected in 2-minute interval from RTMS (remote traffic microwave sensor) in Beijing was used, mainly from the 3rd ring road expressway due to the comparison among the 2 nd, $3 \mathrm{rd}$, and 4 th ring road expressways. The data set consists of rate of flow, speed, and occupancy on workdays from 5:00 to 22:00 during one month from 29 detectors. Discussion for catastrophe focuses on the situations of median lanes and shoulder lanes; the latter ones are often disturbed by vehicles travelling on and off camps. In addition, one direction traffic operation is mainly considered in traffic catastrophe and chaos analysis.

3.1. Traffic Catastrophe Analysis. In our analysis, data characteristics were firstly descripted, and then parameters statistics was analyzed including relation between occupancy and density and flow-density regression was realized; critical catastrophe density calculation was followed mainly on condition of median lane and condition of shoulder lane. The part of traffic chaos analysis consisted of qualitative analysis and quantitative analysis and the maximum Lyapunov exponents on flow, speed, and occupancy were calculated, respectively.

3.1.1. Data Characteristics. In the first step, data cleaning was used on the data set, including correcting abnormal data, adding lost data according to time stamps. Then the data set collected from RTMS no. 3060 could be used for the next steps, and two figures, Figures 1 and 2, are shown as follows based on the data.

Figure 1 illustrates the graphical relationships of speedflow, and it can be inferred from Figure 1 that the free flow phase with high velocity and low rate of flow discontinuously evolves into the congested phase; a discontinuity or jump phenomenon happens through the evolution. Data scatters in the free and congested zones, however, points are sparse

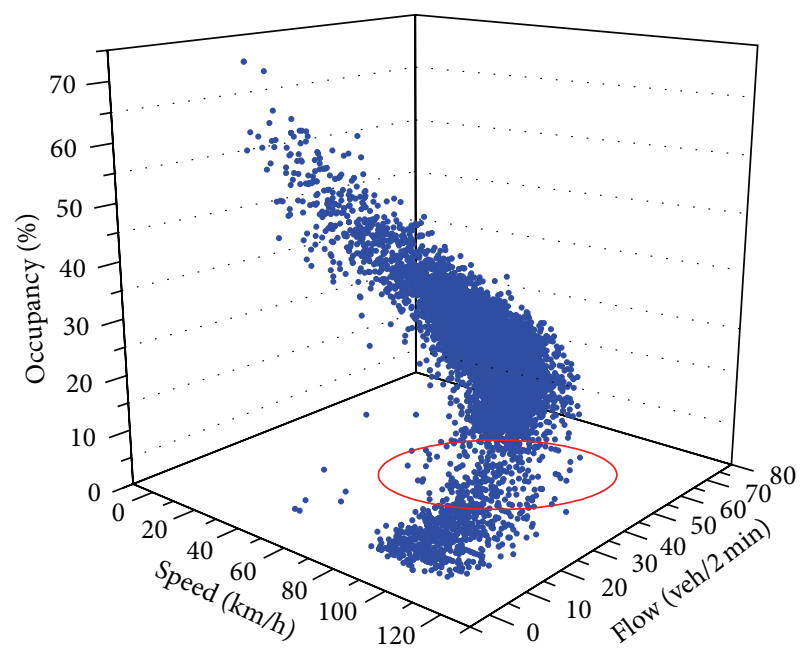

FIGURE 2: Scatter illustration of flow-speed-occupancy from RTMS 3060. Small blue points stand for the traffic characteristics in flow-speed-occupancy space, and the red ellipse separates the free condition with congested condition. Symbols show conditions of discontinuity.

in the joint zone, and the same condition also appears in the Figure 2.

3.1.2. Parameters Statistics. This part includes the relation between occupancy and density; flow-density regression function was also realized.

(a) Relation between Occupancy and Density. Catastrophe critical density was deduced based on the maximum parameters of traffic flow, speed at free flow condition, and maximum congested density; however, only occupancy was recorded in the data, and then it had to be changed to the density. Based on Zhao et al.'s work [27], the relation between occupancy and density was deduced as follows:

$$
\text { density }=\frac{\text { constant } * \text { occupancy }}{\text { leng }_{\text {vehicle }}+\text { leng }_{\text {detector }}} .
$$

Additionally, the average lengths of vehicles, leng vehicle' in the ring road expressway in Beijing were 5.78 meters for median lanes, 6.03 meters for middle lanes, and 6.33 meters for shoulder lanes [27], and the constant in (14) was chosen as 10 for the expressway condition in Beijing. Meanwhile, leng $_{\text {detector }}$ denoting the detect distance along the lane was 2 meters based on the RTMS property. Then the median lanes occupancy-density function and shoulder lanes occupancydensity function are inferred as below:

condition of median lanes:

$$
\text { density }_{\text {median }}=\frac{10 \times \text { occupancy }^{7.78}}{7.7}
$$

condition of shoulder lanes:

$$
\text { density }_{\text {shoulder }}=\frac{10 \times \text { occupancy }}{8.33} .
$$


TABLE 1: Significance test of traffic flow-density quadratic regression model on median lanes.

\begin{tabular}{|c|c|c|c|c|}
\hline \multirow{2}{*}{$R$} & \multirow{2}{*}{\multicolumn{2}{|c|}{$F$ test }} & \multicolumn{2}{|c|}{$t$-test } \\
\hline & & & Quadratic term & Linear term \\
\hline 0.783 & 139201.462 & 0.001 & -475.3320 .001 & 526.5100 .001 \\
\hline
\end{tabular}

TABLE 2: Significance test of traffic flow-density quadratic regression model on shoulder lanes.

\begin{tabular}{|c|c|c|c|c|}
\hline \multirow{2}{*}{$R$} & \multirow{2}{*}{\multicolumn{2}{|c|}{$F$ test }} & \multicolumn{2}{|c|}{$t$-test } \\
\hline & & & Quadratic term & Linear term \\
\hline 0.728 & 98260.656 & 0.000 & -400.4780 .001 & 441.5320 .001 \\
\hline
\end{tabular}

(b) Flow-Density Regression. The quadratic flow-density regression model was built based on the actual data, and the results of parameters and model significance testing were shown as follows.

Quadratic flow-density regression in median lanes was inferred as

$$
Q=-0.01963 \cdot K^{2}+1.892 \cdot K+18.43 .
$$

From Table 1 it can be read that $R=0.783$, and $F$ testing results are smaller than 0.05 ; the relation between $Q$ and $K$ is statistical reliable. In addition, $t$-testing results for regression coefficients are also smaller than 0.05 , which means they are statistical significant.

Quadratic flow-density regression in shoulder lanes was inferred as

$$
Q=-0.01326 \cdot K^{2}+1.389 \cdot K+14.63 .
$$

From Table 2, it is noted that $R=0.728$, and $F$ testing results are smaller than 0.05; the relation between $Q$ and $K$ is statistical authentic. In addition, $t$-testing results for regression coefficients are also smaller than 0.05, which means they are statistical significant.

3.1.3. Critical Catastrophe Density Calculation. Equation (13) was used for calculating critical catastrophe density, and the results of median lanes and shoulder lanes were inferred as follows.

(a) Condition of Median Lane. According to (17), maximum $Q$ can be calculated when $K=48.19 \mathrm{veh} / \mathrm{km}$ and $Q_{\max }=64.02 \mathrm{veh} / 2 \mathrm{~min}$. Free flow velocity value, $V_{f}$, could be inferred from actual data by building speed-flow Greenshields model; then $V_{f}=77.6 \mathrm{~km} / \mathrm{h}$, with congested density $K_{j}=86.52 \mathrm{veh} / \mathrm{km}$. At last, the critical catastrophe density $K_{\text {median }}=23.11 \mathrm{veh} / \mathrm{km}$.

(b) Condition of Shoulder Lane. According to (18), maximum $Q$ can be calculated when $K=52.38 \mathrm{veh} / \mathrm{km}$ and $Q_{\max }=51.01 \mathrm{veh} / 2 \mathrm{~min}$. Free flow velocity value, $V_{f}$, could be inferred from actual data by building speed-flow Greenshields model; then $V_{f}=69.2 \mathrm{~km} / \mathrm{h}$, with congested density $K_{j}=88.54 \mathrm{veh} / \mathrm{km}$. At last, the critical catastrophe density $K_{\text {shoulder }}=22.61 \mathrm{veh} / \mathrm{km}$.
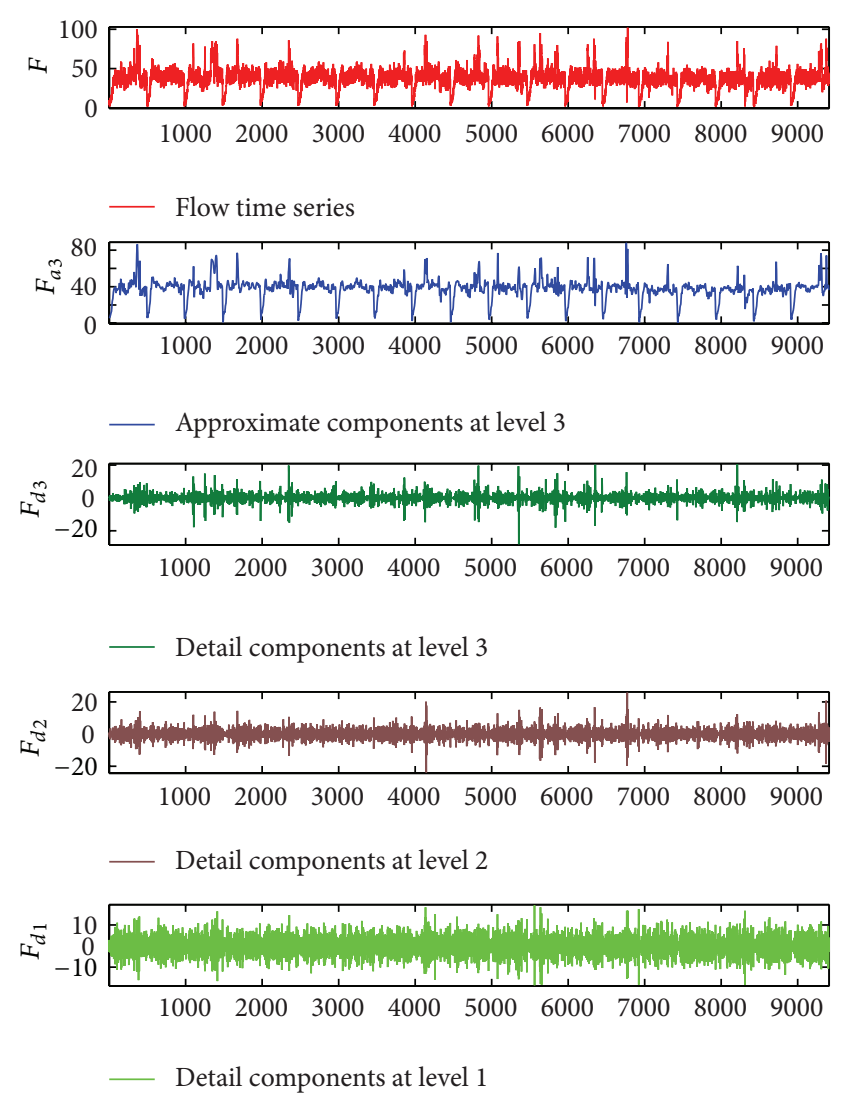

FIgURE 3: Flow time series wavelet decomposition at the 15th detector. Self-similarity is present at each decomposition level in wavelet decomposition diagrams on flow. Symbols qualitatively show the chaos existence in flow time series.

3.2. Traffic Chaos Analysis. It is difficult to build dynamic function considering complex factors in traffic flow system, so the time series analysis will be used. Firstly, wavelet decomposition diagram was used to identify traffic chaos in the qualitative aspect. Then, reconstruction of the phase space with C-C method and calculation of the largest Lyapunov exponent were implemented by Wolf method and small data sets method, respectively, in the quantitative aspect.

The data set consists of flow, speed, and occupancy in 2 minutes from 5:00 to 22:00 collected from 29 detectors located at the 3rd ring road expressway in a month in Beijing. Also, one direction traffic operation is mainly considered.

3.2.1. Qualitative Analysis. Wavelet decomposition diagrams on traffic flow, speed, and occupancy from the location of the 15th detector with DB 4 at level 3 are shown as Figures 3, 4, and 5 .

3.3. Results. It is indicated that components distribute in frequency domain, and self-similarity is present at each decomposition level in wavelet decomposition diagrams on 


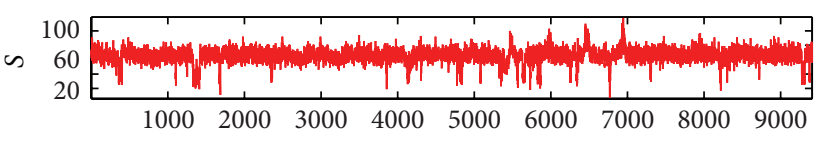

_ Speed time series
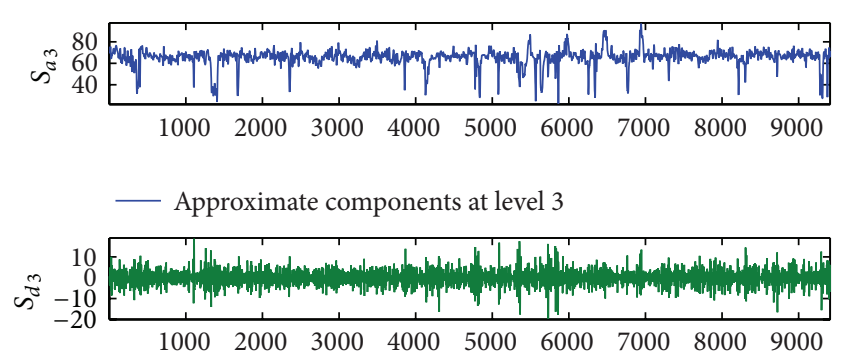

- Detail components at level 3
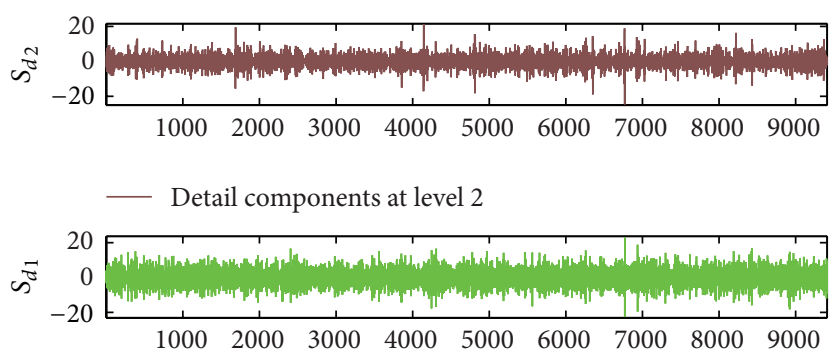

_ Detail components at level 1

FIgURE 4: Speed time series wavelet decomposition at the 15th detector. Self-similarity is present at each decomposition level in wavelet decomposition diagrams on speed. Symbols qualitatively show the chaos existence in speed time series.

flow, speed, and occupancy, which are similar to the characteristics represented in the wavelet decomposition diagrams of chaotic temporal sequence.

3.3.1. Quantitative Analysis. C-C method was applied to calculate the delay time and the embedding dimension to reconstruct the phase space; after that Wolf method and small data sets method were used to calculate the largest Lyapunov exponents for the traffic three parameters. The C-C method results for flow are shown as Figure 6, which are also the detail illustrations for the location of the 15th detector.

The same methods were used to analyze chaotic characteristic for other detectors, and the results on the delay time $\tau$, the embedding dimension $m$, and the largest Lyapunov exponents $\lambda_{\max }$ based on the Wolf method (M1) and small data method (M2) were recorded in Tables 3, 4, and 5.

From Table 3, it can be noted that all the largest Lyapunov exponents are positive in the Wolf method results; meanwhile all the largest Lyapunov exponents from small data sets method are positive except the location of the 26th detector, which need more information to identify chaos. It can be inferred that results from both methods have similar evolution tendency, and they have the largest value at the location of the 7 th detector, as Figure 7 shows.
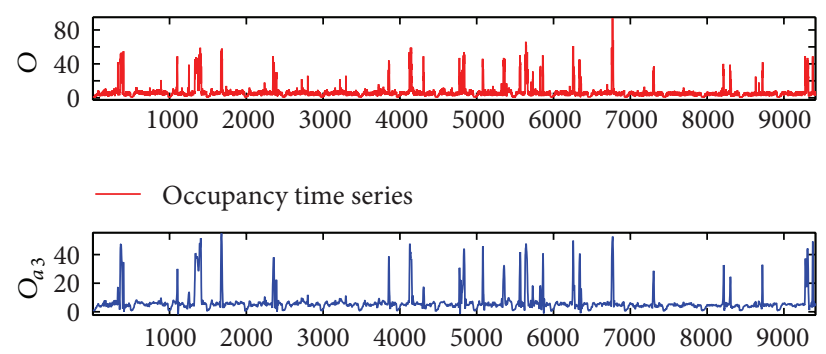

_ Approximate components at level 3
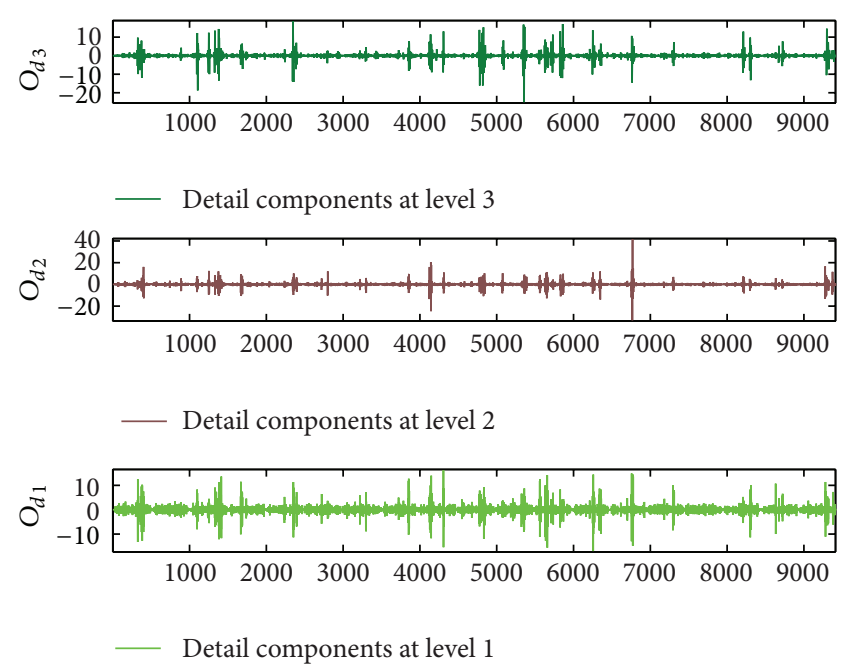

FIGURE 5: Occupancy time series wavelet decomposition at the 15th detector. Self-similarity is present at each decomposition level in wavelet decomposition diagrams on occupancy. Symbols qualitatively show the chaos existence in occupancy time series.
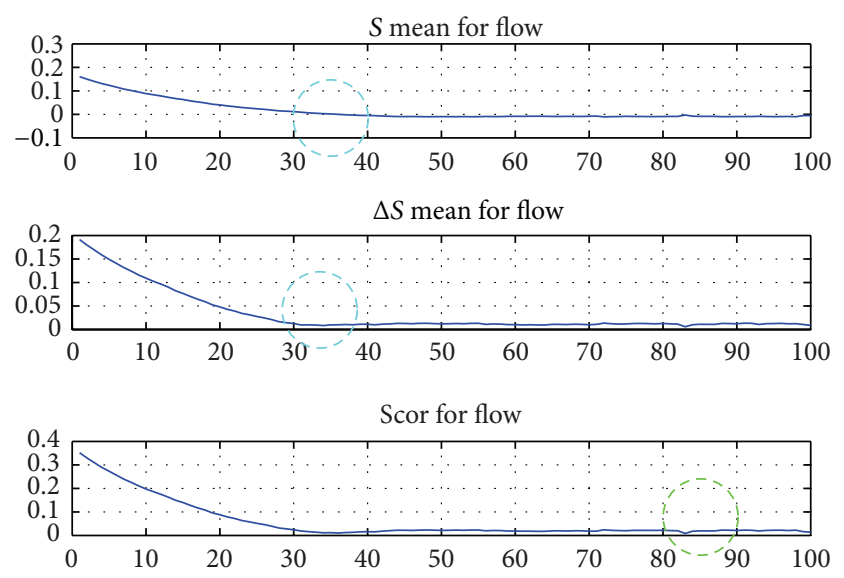

Figure 6: C-C method result for flow time series. According to Kim et al.'s work [26], delay time $\tau_{d}=t \cdot \tau_{s}\left(\tau_{s}=1\right)$ can be deduced from the first zero crossing of $S$ mean or the first local minimum of delta $S$ mean in the blue circle zones, according to the minimum of $S_{\text {cor }}=$ $\tau_{d} \cdot(m-1)$ in the green circle zone; then the integral embedding dimension $m$ can be inferred. 
TABLE 3: C-C method result and Lyapunov exponents for flow.

\begin{tabular}{|c|c|c|c|c|c|c|c|c|}
\hline Detectors no. & 1 & 2 & 3 & 4 & 5 & 6 & 7 & 8 \\
\hline \multicolumn{9}{|l|}{$\mathrm{C}-\mathrm{C}$} \\
\hline$m$ & 4 & 6 & 4 & 2 & 4 & 3 & 3 & 4 \\
\hline$\tau$ & 36 & 22 & 35 & 36 & 32 & 40 & 42 & 36 \\
\hline \multicolumn{9}{|l|}{$\lambda_{\max }$} \\
\hline M1 & 0.461 & 0.546 & 0.208 & 0.807 & 0.552 & 0.811 & 1.038 & 0.315 \\
\hline M2 & 0.013 & 0.009 & 0.022 & 0.015 & 0.041 & 0.068 & 0.068 & 0.032 \\
\hline Detectors no. & 9 & 10 & 11 & 12 & 13 & 14 & 15 & 16 \\
\hline \multicolumn{9}{|l|}{$\mathrm{C}-\mathrm{C}$} \\
\hline$m$ & 3 & 3 & 3 & 4 & 4 & 4 & 4 & 4 \\
\hline$\tau$ & 35 & 37 & 34 & 24 & 36 & 30 & 31 & 38 \\
\hline \multicolumn{9}{|l|}{$\lambda_{\max }$} \\
\hline M1 & 0.804 & 0.492 & 0.767 & 0.398 & 0.323 & 0.244 & 0.29 & 0.339 \\
\hline M2 & 0.019 & 0.028 & 0.049 & 0.065 & 0.018 & 0.027 & 0.032 & 0.023 \\
\hline Detectors no. & 17 & 18 & 19 & 20 & 21 & 22 & 23 & 24 \\
\hline \multicolumn{9}{|l|}{$\mathrm{C}-\mathrm{C}$} \\
\hline$m$ & 8 & 4 & 3 & 4 & 3 & 3 & 4 & 3 \\
\hline$\tau$ & 13 & 32 & 47 & 33 & 34 & 34 & 31 & 36 \\
\hline \multicolumn{9}{|l|}{$\lambda_{\max }$} \\
\hline M1 & 0.117 & 0.139 & 0.696 & 0.287 & 0.4 & 0.592 & 0.177 & 0.609 \\
\hline M2 & 0.007 & 0.018 & 0.031 & 0.019 & 0.022 & 0.025 & 0.032 & 0.037 \\
\hline Detectors no. & 25 & 26 & 27 & 28 & 29 & - & - & - \\
\hline \multicolumn{9}{|l|}{ C-C } \\
\hline$m$ & 4 & 49 & 4 & 2 & 3 & - & - & - \\
\hline$\tau$ & 37 & 2 & 31 & 32 & 35 & - & - & - \\
\hline \multicolumn{9}{|l|}{$\lambda_{\max }$} \\
\hline M1 & 0.498 & 0.008 & 0.261 & 0.495 & 0.282 & - & - & - \\
\hline M2 & 0.012 & -0.01 & 0.023 & 0.027 & 0.029 & - & - & - \\
\hline
\end{tabular}

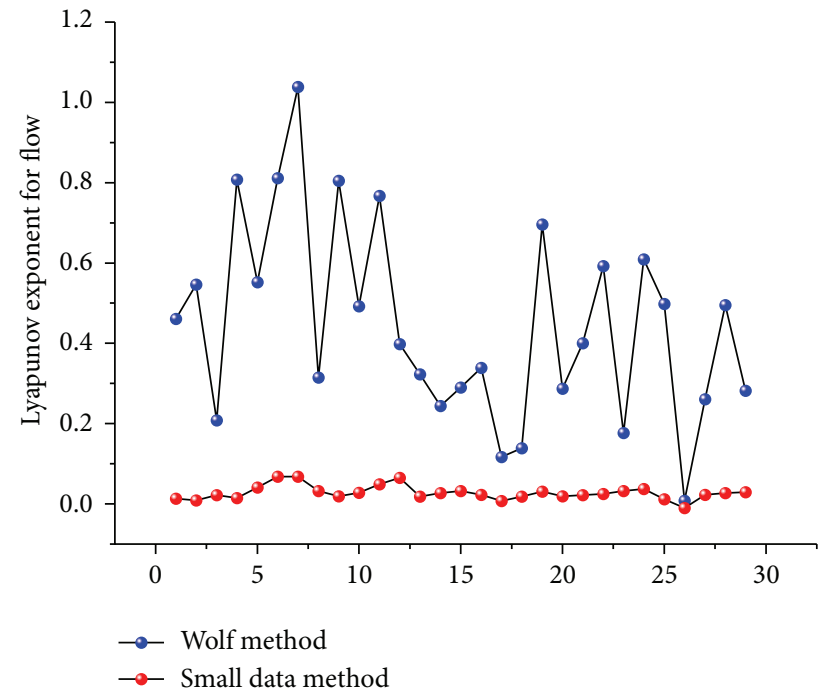

FIgURE 7: The largest Lyapunov exponents of flow time series from 29 detectors. Results for flow temporal sequence by both methods show that the exponents are positive except at the 26th detector and values at the 7 th detector location are larger than those at other locations in the spatial sequence.

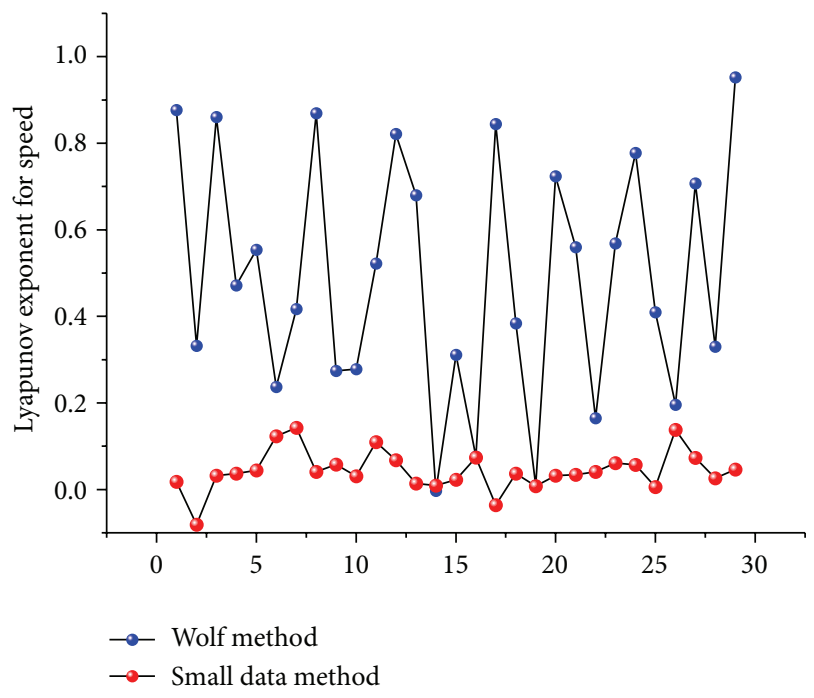

FIGURE 8: The largest Lyapunov exponents of speed time series from 29 detectors. Values are positive except those at the 14th detector by Wolf method, and values at the 2 nd and 17 th detectors are negative by small data sets method. 
TABLE 4: C-C method result and Lyapunov exponents for speed.

\begin{tabular}{|c|c|c|c|c|c|c|c|c|}
\hline Detector no. & 1 & 2 & 3 & 4 & 5 & 6 & 7 & 8 \\
\hline \multicolumn{9}{|l|}{$\mathrm{C}-\mathrm{C}$} \\
\hline$m$ & 3 & 7 & 3 & 4 & 4 & 4 & 3 & 3 \\
\hline$\tau$ & 29 & 8 & 38 & 34 & 30 & 28 & 43 & 31 \\
\hline \multicolumn{9}{|l|}{$\lambda_{\max }$} \\
\hline M1 & 0.876 & 0.332 & 0.86 & 0.472 & 0.554 & 0.237 & 0.417 & 0.869 \\
\hline M2 & 0.018 & -0.081 & 0.032 & 0.037 & 0.044 & 0.123 & 0.143 & 0.041 \\
\hline Detector no. & 9 & 10 & 11 & 12 & 13 & 14 & 15 & 16 \\
\hline \multicolumn{9}{|l|}{$\mathrm{C}-\mathrm{C}$} \\
\hline$m$ & 4 & 4 & 3 & 3 & 5 & 96 & 5 & 5 \\
\hline$\tau$ & 33 & 31 & 53 & 55 & 24 & 1 & 24 & 22 \\
\hline \multicolumn{9}{|l|}{$\lambda_{\max }$} \\
\hline M1 & 0.274 & 0.278 & 0.522 & 0.821 & 0.68 & -0.003 & 0.311 & 0.076 \\
\hline M2 & 0.058 & 0.031 & 0.11 & 0.068 & 0.014 & 0.009 & 0.023 & 0.074 \\
\hline Detector no. & 17 & 18 & 19 & 20 & 21 & 22 & 23 & 24 \\
\hline \multicolumn{9}{|l|}{$\mathrm{C}-\mathrm{C}$} \\
\hline$m$ & 6 & 4 & 89 & 3 & 4 & 4 & 3 & 3 \\
\hline$\tau$ & 6 & 25 & 1 & 25 & 28 & 30 & 43 & 51 \\
\hline \multicolumn{9}{|l|}{$\lambda_{\max }$} \\
\hline M1 & 0.844 & 0.384 & 0.009 & 0.723 & 0.56 & 0.165 & 0.569 & 0.777 \\
\hline M2 & -0.036 & 0.037 & 0.008 & 0.032 & 0.034 & 0.041 & 0.061 & 0.057 \\
\hline Detector no. & 25 & 26 & 27 & 28 & 29 & - & - & - \\
\hline \multicolumn{9}{|l|}{$\overline{C-C}$} \\
\hline$m$ & 6 & 3 & 3 & 3 & 3 & - & - & - \\
\hline$\tau$ & 15 & 28 & 39 & 34 & 40 & - & - & - \\
\hline \multicolumn{9}{|l|}{$\lambda_{\max }$} \\
\hline M1 & 0.41 & 0.196 & 0.707 & 0.33 & 0.952 & - & - & - \\
\hline M2 & 0.006 & 0.138 & 0.073 & 0.026 & 0.046 & - & - & - \\
\hline
\end{tabular}

From Table 4, it can be indicated that all the largest Lyapunov exponents are positive except the location of 14th detector in the Wolf method results; meanwhile all the largest Lyapunov exponents from small data sets method are positive except those at the locations of 2 nd and 17 th detectors, which need more information to identify chaos. The spatial evolution tendency is illustrated as Figure 8.

From Table 5, it can be inferred that all the largest Lyapunov exponents are positive in the Wolf method results; meanwhile all the largest Lyapunov exponents from small data sets method are positive except those at the location of 2nd detector, which need more information to identify chaos. The spatial evolution tendency is shown as Figure 9.

This part consists of data analysis results on traffic catastrophic and chaotic characteristic.

3.3.2. Traffic Flow Catastrophe Analysis Results. From the calculation results, it can be indicated that critical density is smaller than maximum-flow density and congested density. And from the aspect of data description it is indicated that when the density value reached the critical value, the traffic flow phase would transform from unconstrained to constrained with the jump phenomenon. So when the traffic operation is under monitor, once the density arises to the critical level, several control measures should be actualized to avoid severe congestion, and the traffic volume of travelling on-off ramps should be reduced by route guidance in order to keep a safe driving zone and avoid traffic accidents.

The critical density level in different lanes has different values, and the results demonstrate that catastrophe density in median lanes $(23.11 \mathrm{veh} / \mathrm{km})$ is larger than that in shoulder lanes $(22.61 \mathrm{veh} / \mathrm{km})$, and that means conditions of median lanes could be transferred into the catastrophe phase later than the conditions of shoulder lanes in terms of density. Vehicles travelling on or off the ramps will change lanes across the shoulder lanes, which may cause the jump phenomenon earlier than median lanes in terms of density. When traffic flow is disturbed by certain inducements, density will be changed as a wave spreads backward with certain speed, and it is the "shock wave" that causes the flow-density catastrophe, especially in shoulder lanes near ramps. For example, when vehicle drives across the shoulder lanes from the ramps, the back ones' normal driving will be influenced by this, and local density may be increased and causes block.

3.3.3. Traffic Flow Chaotic Analysis Results. Table 6 is inferred from the largest Lyapunov exponent results. And the situation at the 2 nd detector shows that the largest Lyapunov exponents 
TABLE 5: C-C method result and Lyapunov exponents for occupancy.

\begin{tabular}{|c|c|c|c|c|c|c|c|c|}
\hline Detector no. & 1 & 2 & 3 & 4 & 5 & 6 & 7 & 8 \\
\hline \multicolumn{9}{|l|}{$\mathrm{C}-\mathrm{C}$} \\
\hline$m$ & 3 & 15 & 3 & 3 & 4 & 4 & 5 & 4 \\
\hline$\tau$ & 40 & 6 & 35 & 44 & 35 & 28 & 28 & 33 \\
\hline \multicolumn{9}{|l|}{$\lambda_{\max }$} \\
\hline M1 & 0.534 & 0.046 & 0.548 & 0.447 & 0.381 & 0.394 & 0.248 & 0.342 \\
\hline M2 & 0.022 & -0.034 & 0.054 & 0.072 & 0.074 & 0.094 & 0.078 & 0.067 \\
\hline Detector no. & 9 & 10 & 11 & 12 & 13 & 14 & 15 & 16 \\
\hline \multicolumn{9}{|l|}{ C-C } \\
\hline$m$ & 3 & 3 & 5 & 3 & 98 & 3 & 5 & 4 \\
\hline$\tau$ & 45 & 34 & 25 & 41 & 1 & 45 & 14 & 27 \\
\hline \multicolumn{9}{|l|}{$\lambda_{\max }$} \\
\hline M1 & 0.629 & 0.552 & 0.219 & 0.769 & 0.002 & 0.396 & 0.155 & 0.161 \\
\hline M2 & 0.067 & 0.059 & 0.085 & 0.114 & 0.027 & 0.08 & 0.061 & 0.068 \\
\hline Detector no. & 17 & 18 & 19 & 20 & 21 & 22 & 23 & 24 \\
\hline \multicolumn{9}{|l|}{$\mathrm{C}-\mathrm{C}$} \\
\hline$m$ & 100 & 5 & 99 & 3 & 4 & 3 & 4 & 3 \\
\hline$\tau$ & 1 & 25 & 1 & 35 & 35 & 18 & 30 & 44 \\
\hline \multicolumn{9}{|l|}{$\lambda_{\max }$} \\
\hline M1 & 0.002 & 0.195 & 0.001 & 0.281 & 0.282 & 0.288 & 0.258 & 0.564 \\
\hline M2 & 0.014 & 0.046 & 0.009 & 0.056 & 0.053 & 0.044 & 0.043 & 0.063 \\
\hline Detector no. & 25 & 26 & 27 & 28 & 29 & - & - & - \\
\hline \multicolumn{9}{|l|}{$\mathrm{C}-\mathrm{C}$} \\
\hline$m$ & 7 & 97 & 3 & 3 & 3 & - & - & - \\
\hline$\tau$ & 16 & 1 & 59 & 34 & 46 & - & - & - \\
\hline \multicolumn{9}{|l|}{$\lambda_{\max }$} \\
\hline M1 & 0.055 & 0.001 & 0.669 & 0.203 & 0.297 & - & - & - \\
\hline M2 & 0.019 & 0.008 & 0.074 & 0.042 & 0.055 & - & - & - \\
\hline
\end{tabular}

of speed and occupancy time series are negative from the small data sets method and the largest Lyapunov exponents of flow are near zero. The chaotic characteristic at 2 nd detector is not supported.

The largest Lyapunov exponent of speed time series at the 14 th detector is negative.

The largest Lyapunov exponent of speed time series at the 17th detector is negative, the largest Lyapunov exponent of flow time series is nearly equal to zero from the small data sets method, and the largest Lyapunov exponent of occupancy time series is nearly equal to zero from the Wolf method.

The largest Lyapunov exponent of flow time series at the 26th detector is negative from the small data sets method, and the largest Lyapunov exponent of occupancy time series is nearly equal to zero from the Wolf method.

Traffic chaotic characteristic are not obvious in these 4 locations, and that needs further discussion and may be periodic or stochastic.

\section{Conclusions}

Traffic flow data collected from the 3rd ring road expressway in Beijing was applied to develop flow-density model and speed-density Greenshields model. Then the density was discussed based on the traffic wave speed function with cusp catastrophe theory, which was used to analyze the traffic nonlinear characteristic in freeway. Results show that the critical density was smaller than maximum-flow density and congested density. Especially, density conditions between median lanes and shoulder lanes were deeply discussed, and the results indicated that the critical catastrophe density value in the median lanes was a little higher than that in the shoulder lanes. It is important to control travelling on-off ramps when the density reaches the critical level.

Traffic chaotic characteristics are observed obviously; the C-C method was applied to reconstruct phase space; Wolf method and small data method sets were used to estimate the largest Lyapunov exponents; respectively, the results indicated that data collected at 25 of 29 detectors were positive implying an exponential divergence of the trajectories and a strong signature of chaos. Distinguishing between chaotic and stochastic behaviors might be discussed in the later research.

This paper mainly focused on the actual data analysis in condition of the 3rd ring road expressway in Beijing, and the result may be limited by the collected data precision with initial time interval which may contain noise and cause fluctuations in the calculation in practice; the quantitative 


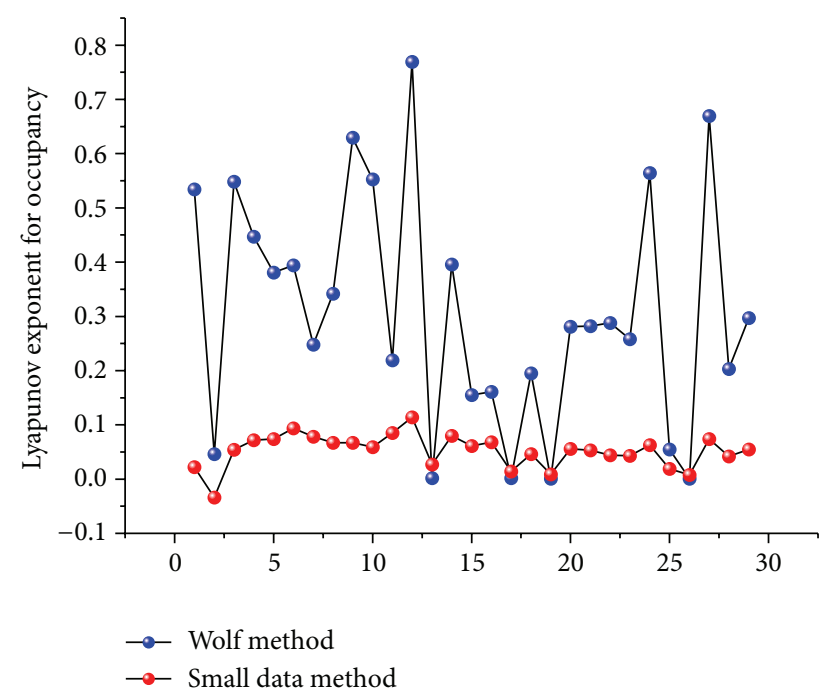

Figure 9: The largest Lyapunov exponents of occupancy time series from 29 detectors. Results from Wolf method are positive, and only value at 2 nd detectors is negative by small data method.

TABLE 6: Uncertainty conditions from chaotic analysis results.

\begin{tabular}{|c|c|c|c|c|}
\hline \multirow{2}{*}{ Detector no. } & \multicolumn{2}{|c|}{$\mathrm{C}-\mathrm{C}$ results } & \multicolumn{2}{|c|}{$\lambda_{\max }$} \\
\hline & $\tau$ & $m$ & M1 & M2 \\
\hline \multicolumn{5}{|l|}{2} \\
\hline Flow & 6 & 22 & 0.546 & 0.009 \\
\hline Speed & 7 & 8 & 0.332 & -0.081 \\
\hline Occupancy & 15 & 6 & 0.046 & -0.034 \\
\hline \multicolumn{5}{|l|}{14} \\
\hline Flow & 4 & 30 & 0.244 & 0.027 \\
\hline Speed & 96 & 1 & -0.003 & 0.009 \\
\hline Occupancy & 3 & 45 & 0.396 & 0.080 \\
\hline \multicolumn{5}{|l|}{17} \\
\hline Flow & 8 & 13 & 0.117 & 0.007 \\
\hline Speed & 6 & 6 & 0.844 & -0.036 \\
\hline Occupancy & 100 & 1 & 0.002 & 0.014 \\
\hline \multicolumn{5}{|l|}{26} \\
\hline Flow & 49 & 2 & 0.008 & -0.010 \\
\hline Speed & 3 & 28 & 0.196 & 0.138 \\
\hline Occupancy & 97 & 1 & 0.001 & 0.008 \\
\hline
\end{tabular}

analysis of chaotic systems especially is usually sensitive to not only the data size, but also the observation time. According to the results, three kinds of traffic parameters time series represent different chaotic characteristics at the same location; a certain exclusive model may be needed to identify the chaos with multidimension analysis based on traffic parameters; meanwhile, multi-interval analysis and spatial factors will be considered in new models in the proceeding research, and management and control strategy, that is, designing signal, ramp metering, or speed controlling, should be yielded to avoid traffic congestion and even the accidents in the later research. The relation between the catastrophe and chaos based on the bifurcation theory needs more demonstration in the future research.

\section{Conflict of Interests}

The authors declare that there is no conflict of commercial or associative interests regarding the publication of this work.

\section{Acknowledgments}

This work is supported by Jiangsu Province University Graduate Student Research and Innovation Program no. CXLX13_109 and the National Natural Science Foundation of China under Grant no. 61374195. The authors would like to thank the anonymous referees for their valuable criticism and fruitful comments.

\section{References}

[1] M. Perc, "Premature seizure of traffic flow due to the introduction of evolutionary games," New Journal of Physics, vol. 9, article A3, 2007.

[2] M. Perc and A. Szolnoki, "Coevolutionary games-a mini review," BioSystems, vol. 99, no. 2, pp. 109-125, 2010.

[3] M. Perc, J. Gómez-Gardeñes, A. Szolnoki, L. M. Floría, and Y. Moreno, "Evolutionary dynamics of group interactions on structured populations: a review," Journal of the Royal Society: Interface, vol. 10, no. 80, Article ID 20120997, 2013.

[4] M. Carey, H. Bar-Gera, D. Watling, and C. Balijepalli, "Implementing first-in-first-out in the cell transmission model for networks," Transportation Research B: Methodological, vol. 65, pp. 105-118, 2014.

[5] N. Zheng, R. A. Waraich, K. W. Axhausen, and N. Geroliminis, "A dynamic cordon pricing scheme combining the Macroscopic Fundamental Diagram and an agent-based traffic model," Transportation Research Part A: Policy and Practice, vol. 46, no. 8, pp. 1291-1303, 2012.

[6] S. Tadaki, K. Nishinari, M. Kikuchi, Y. Sugiyama, and S. Yukawa, "Analysis of congested flow at the upper stream of a tunnel," Physica A: Statistical Mechanics and its Applications, vol. 315, no. 1-2, pp. 156-162, 2002.

[7] N. Geroliminis and C. F. Daganzo, "Existence of urban-scale macroscopic fundamental diagrams: some experimental findings," Transportation Research Part B: Methodological, vol. 42, no. 9, pp. 759-770, 2008.

[8] N. Geroliminis and J. Sun, "Properties of a well-defined macroscopic fundamental diagram for urban traffic," Transportation Research Part B: Methodological, vol. 45, no. 3, pp. 605-617, 2011.

[9] C. F. Daganzo, V. V. Gayah, and E. J. Gonzales, "Macroscopic relations of urban traffic variables: bifurcations, multivaluedness and instability," Transportation Research B: Methodological, vol. 45, no. 1, pp. 278-288, 2011.

[10] F. L. Hall, "An interpretation of speed-flow-concentration relationships using catastrophe theory," Transportation Research Part A: General, vol. 21, no. 3, pp. 191-201, 1987.

[11] G. J. Forbes and F. L. Hall, "The applicability of catastrophe theory in modelling freeway traffic operations," Transportation Research A: General, vol. 24, no. 5, pp. 335-344, 1990.

[12] J. A. Acha-Daza and F. L. Hall, "Application of catastrophe theory to traffic flow variables," Transportation Research B, vol. 28, no. 3, pp. 235-250, 1994. 
[13] Y. P. Zhang and Y. L. Pei, "The application of cusp catastrophe theory in traffic flow prediction," in Proceedings of the IEEE Intelligent Transportation Systems, vol. 1, pp. 628-631, October 2003.

[14] J. Guo, X. L. Chen, and H. Z. Jin, "Based on the cusp catastrophe to research the relationship among traffic flow three parameters," in Proceedings of the IEEE International Conference on Automation and Logistics (ICAL '07), pp. 858-861, August 2007.

[15] A. E. Papacharalampous and E. I. Vlahogianni, "Modeling microscopic freeway traffic using cusp catastrophe theory," IEEE Intelligent Transportation Systems Magazine, vol. 6, no. 1, pp. 6-16, 2014.

[16] X. Zhang and D. F. Jarrett, "Stability analysis of the classical carfollowing model," Transportation Research B: Methodological, vol. 31, no. 6, pp. 441-462, 1997.

[17] T. Nagatani, "Chaotic jam and phase transition in traffic flow with passing," Physical Review E, vol. 60, no. 2, pp. 1535-1541, 1999.

[18] T. Li, "Nonlinear dynamics of traffic jams," Physica D: Nonlinear Phenomena, vol. 207, no. 1-2, pp. 41-51, 2005.

[19] P. J. Shang, X. W. Li, and S. Kamae, "Chaotic analysis of traffic time series," Chaos, Solitons and Fractals, vol. 25, no. 1, pp. 121128, 2005.

[20] S.-C. Lo and H.-J. Cho, "Chaos and control of discrete dynamic traffic model," Journal of the Franklin Institute, vol. 342, no. 7, pp. 839-851, 2005.

[21] L. W. Lan, J.-B. Sheu, and Y.-S. Huang, "Investigation of temporal freeway traffic patterns in reconstructed state spaces," Transportation Research C: Emerging Technologies, vol. 16, no. 1, pp. 116-136, 2008.

[22] A. D. May Jr. and H. E. M. Keller, "Non-Integer car following models," Highway Research Record, no. 199, pp. 19-32, 1967.

[23] Z. H. Liu, "Chaotic time series analysis," Mathematical Problems in Engineering, vol. 2010, Article ID 720190, 31 pages, 2010.

[24] A. Wolf, J. B. Swift, H. L. Swinney, and J. A. Vastano, "Determining Lyapunov exponents from a time series," Physica D: Nonlinear Phenomena, vol. 16, no. 3, pp. 285-317, 1985.

[25] M. T. Rosenstein, J. J. Collins, and C. J. de Luca, "A practical method for calculating largest Lyapunov exponents from small data sets," Physica D: Nonlinear Phenomena, vol. 65, no. 1-2, pp. 117-134, 1993.

[26] H. S. Kim, R. Eykholt, and J. D. Salas, "Nonlinear dynamics, delay times, and embedding windows," Physica D: Nonlinear Phenomena, vol. 127, no. 1-2, pp. 48-60, 1999.

[27] N. L. Zhao, L. Yu, H. Zhao, J. F. Guo, and H. M. Wen, "Analysis of traffic flow characteristics on ring road expressways in Beijing," Transportation Research Record, vol. 2124, pp. 178-185, 2009. 


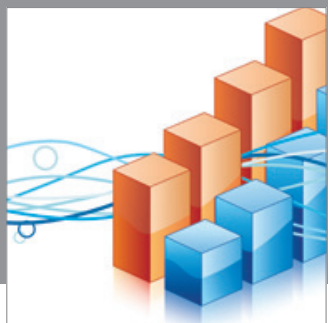

Advances in

Operations Research

mansans

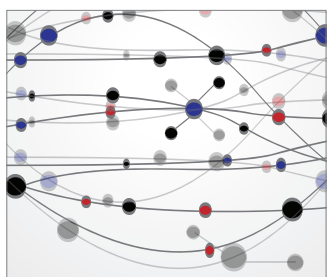

The Scientific World Journal
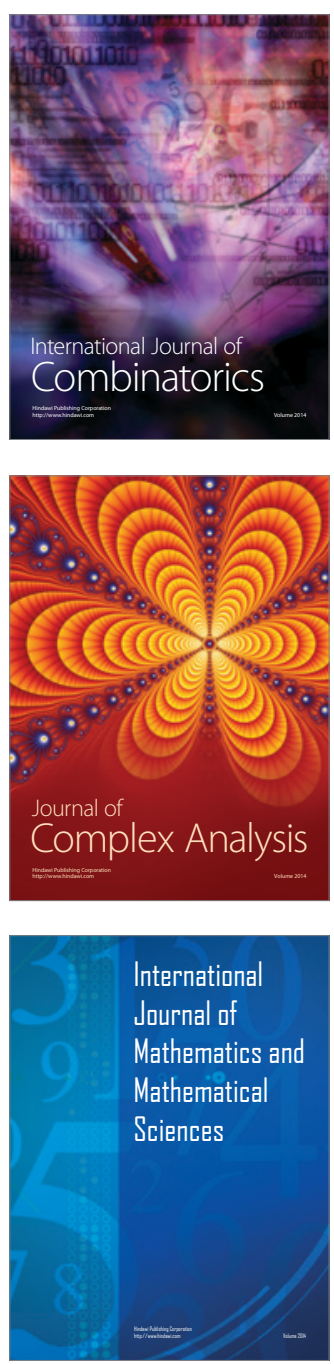
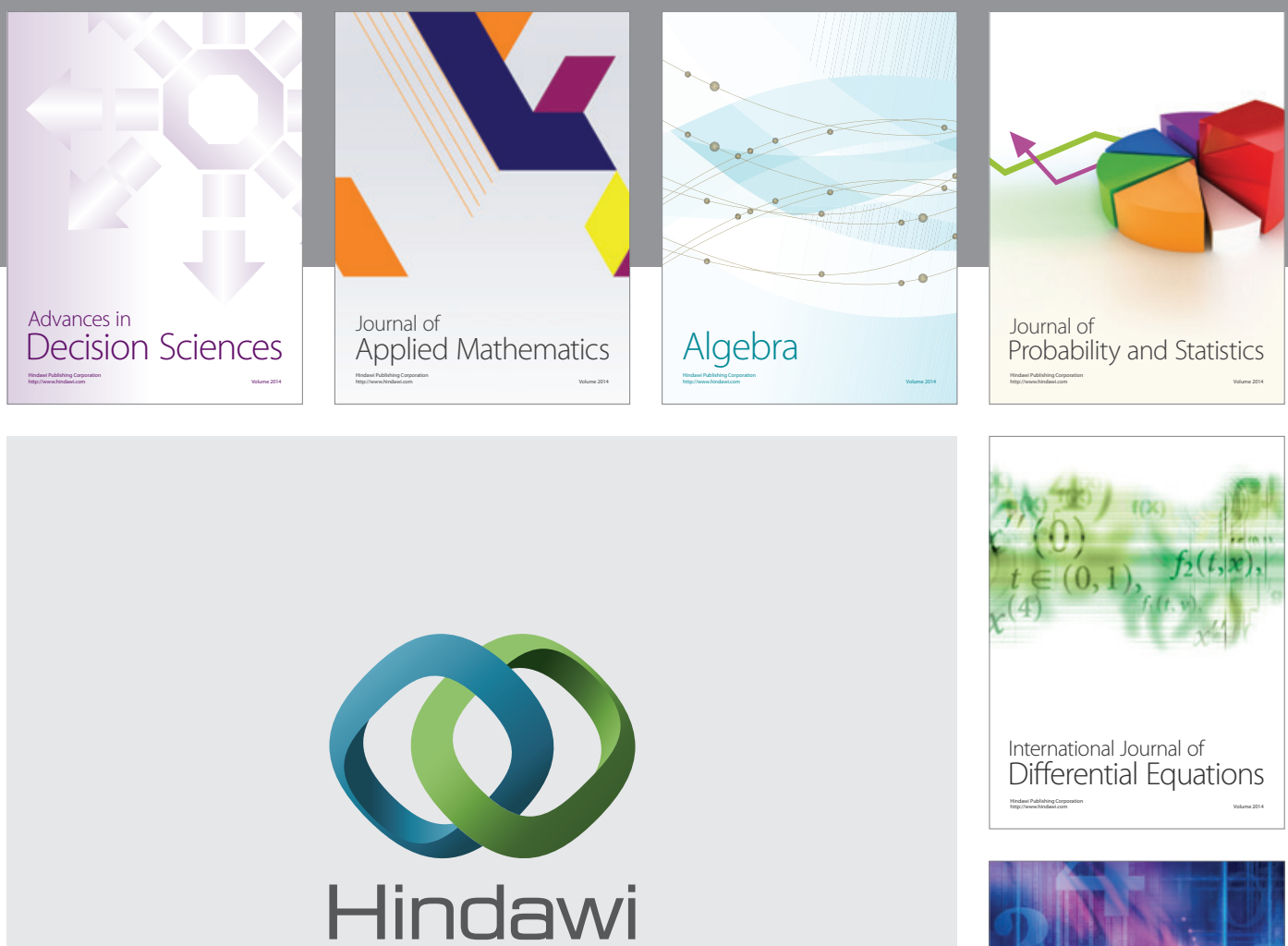

Submit your manuscripts at http://www.hindawi.com
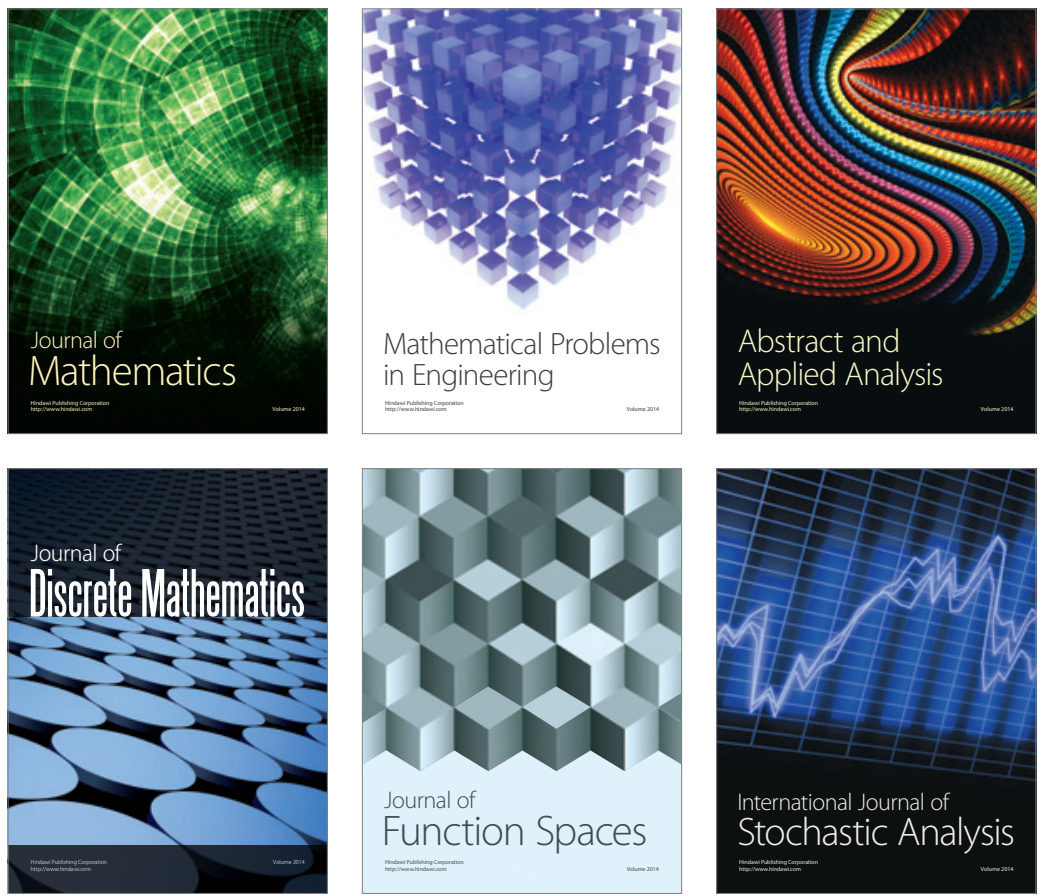

Journal of

Function Spaces

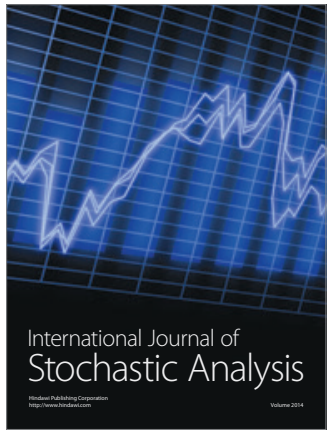

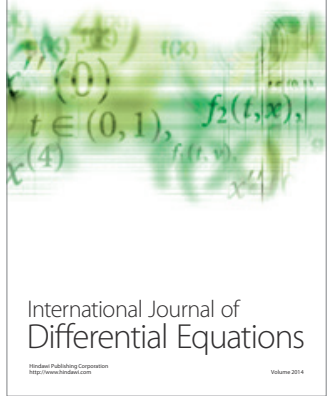
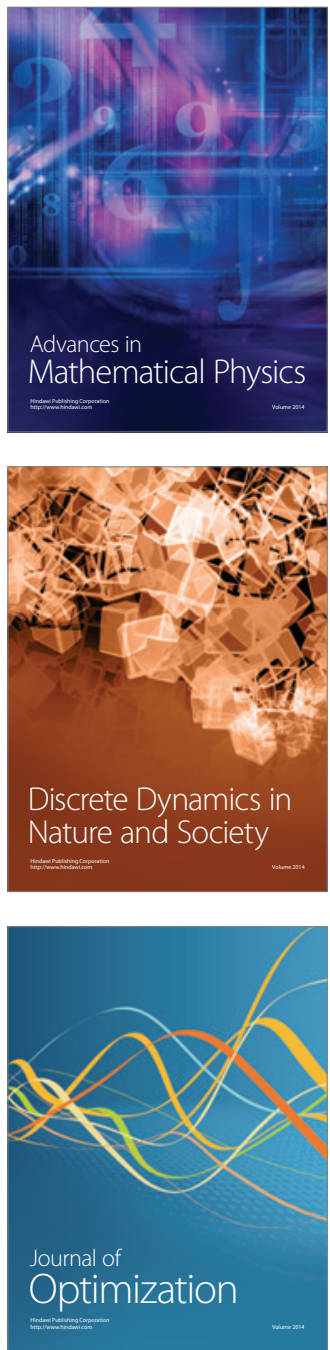